\title{
Mapping shallow soil moisture profiles at the field scale using full-waveform inversion of ground penetrating radar data
}

\author{
Julien Minet*1, Agung Wahyudi ${ }^{1}$, Patrick Bogaert ${ }^{1}$, Marnik Vanclooster ${ }^{1}$, and Sébastien \\ Lambot $^{1,2}$ \\ ${ }^{1}$ Earth and Life Institute, Université catholique de Louvain, Croix du Sud 2 BP 2, B-1348 \\ Louvain-la-Neuve, Belgium \\ ${ }^{2}$ Agrosphere (ICG-4), Institute of Chemistry and Dynamics of the Geosphere, \\ Forschungszentrum Jülich GmbH, D-52425 Jülich, Germany
}

\begin{abstract}
Full-waveform inversions were applied to retrieve surface, two-layered and continuous soil moisture profiles from ground penetrating radar (GPR) data acquired in an 11-ha agricultural field situated in the loess belt area in central Belgium. The radar system consisted of a vector network analyzer combined with an off-ground horn antenna operating in the frequency range 200$2000 \mathrm{MHz}$. The GPR system was computer controlled and synchronized with a differential GPS for real-time data acquisition. Several inversion strategies were also tested using numerical experiments, which in particular demonstrated the potentiality to reconstruct simplified two-layered configurations from more complex, continuous dielectric profiles as prevalent in the environment. The surface soil moisture map obtained assuming a onelayered model showed a global moisture pattern mainly explained by the topography while local moisture patterns indicate a line effect. Two-layered and profile inversions provided consistent estimates with respect to each other and field observations, showing significant moisture increases with depth. However, some discrepancies were observed between the measured and modeled GPR data in the higher frequency ranges, mainly due to surface roughness effects which are not accounted for. The proposed GPR method and inversion strategies show great promise for high-resolution, realtime mapping of soil moisture at the field scale.
\end{abstract}

\footnotetext{
*julien.minet@uclouvain.be
}

\section{Introduction}

Soil moisture dynamics is a key component in many researches and applications like precision agriculture, hydrological studies, meteorological and climatological modeling and other environmental studies. In hydrology, soil moisture is a highly sensitive state-variable in runoff, solute transport, evaporation and erosion processes, as it governs the partitioning between runoff and infiltration, and reducing its uncertainty largely improves modeling precision [Zehe et al., 2005]. In global circulation models, soil moisture highly controls the energy fluxes between the land surface and the atmosphere [Schumann et al., 2009].

Recent developments in microwave remote sensing of surface soil moisture bring increasing opportunities for extensive soil moisture characterization at different spatial and temporal scales, as new remote sensing data products (e.g., from SMOS and SMAP) become available [Wagner et al., 2007]. Nevertheless, a poor agreement still exists between remote sensing derived soil moisture and ground measurements (i.e., gravimetric sampling, time domain reflectometry measurements). Ground-based soil moisture measurement techniques may fail to match the remote sensing retrievals as a result of the different supports of the techniques, particularly with respect to the depth of characterization, as it was stated by Stevens et al. [2008]. In addition, the inherent large spatial variability of soil moisture within a remote sensing pixel implies that a large number of ground measurements must be collected to adequately compare the data. Hence, no absolute relation 
between the backscattered signals from remote sensing sensors and the surface soil moisture exists, necessitating site-specific calibrations [D'Urso and Minacapilli, 2006, Verhoest et al., 2008].

Furthermore, the value of remotely-sensed surface soil moisture may be limited by a lack of correlation between surface and subsurface soil moisture [Vereecken et al., 2008]. As it is directly exposed to atmospheric forcing, surface soil moisture dynamics is a lot more active than subsurface soil moisture. A physical decoupling between surface and subsurface soil moisture may occur considering a wet soil subject to fast evaporation or the propagation of a wetting front in a dry soil, especially in coarse materials. In addition, pedogenetic processes and agricultural practices may lead to varying soil moisture conditions, according to the different soil layer properties [Schaap et al., 2003]. Surface soil moisture may therefore fail to reflect the soil moisture conditions in the subsurface that are actually of interest for a lot of processes [Capehart and Carlson, 1997]. Some studies have addressed this issue in remote sensing acquisition, using transfer functions based on statistical relationships or physically-based hydrodynamic models to relate the soil moisture profile to the remotely-sensed surface soil moisture [Wagner et al., 1999, Ceballos et al., 2005]. Nevertheless, soil moisture profile information cannot be inherently inferred from the single-frequency satellite sensors.

In that respect, ground penetrating radar (GPR) has shown further potentialities to increase the extraction of information about surface and subsurface soil moisture [Huisman et al., 2003, Galagedara et al., 2005, Serbin and Or, 2005, Lunt et al., 2005, Doolittle et al., 2006, Lambot et al., 2008a]. Characterization of soil moisture in multilayered media using inversion of GPR data was performed by Lambot et al. [2004b], van der Kruk [2006] and Strobbia and Cassiani [2007]. In particular, borehole GPR applications can accurately reconstruct 2-D images (tomograms) of the complete soil moisture profile between borehole locations [Binley et al., 2001, Looms et al., 2008], but these techniques remain limited at small-scale (a few meters) studies, as it requires the installation of vertical wells into the soil. Hence, although they showed a good accuracy [e.g., van der Kruk, 2006], these techniques remain largely cumbersome and time-consuming, hampering for the mapping of large areas. Surface soil moisture determination by the surface reflection coefficient method, using offground GPR antennas, have shown a potential for proximal soil moisture sensing at a much larger scale com- pared to the borehole methods [Redman et al., 2002, Serbin and Or, 2003, 2005]. However, this method still remains unused in real field applications due to several practical and theoretical limitations.

Overcoming these drawbacks, the off-ground GPR system developed by Lambot et al. [2004b] has shown promising capabilities for proximal soil moisture sensing at the field scale. The method resorts to fullwaveform forward and inverse modeling of the GPR signal, which inherently maximizes information retrieval capabilities from the radar data in terms of quantity and accuracy. Specific inversion strategies have been developed for the retrieval of soil surface dielectric permittivity and correlated water content [Lambot et al., 2006] and have been applied to field data [Lambot et al., 2008b]. In addition, this ground-based GPR can provide high-resolution soil moisture maps, bridging the scale gap between small-scale invasive measurement techniques and spaceborne sensors.

Following Lambot et al. [2004b] and Minet et al. [2010], we propose to investigate the retrieval of soil moisture profiles by full-waveform inversion of GPR data acquired in an 11-ha agricultural field. The field was situated in the loess belt region in central Belgium (Walhain), consisting mainly of loamy soils. Soil moisture conditions are described by three models, i.e., a one-layered, a two-layered and a continuously-variable profile model. Numerical experiments are first presented, that evaluate GPR inversions assuming the twolayered soil model facing continuous soil moisture profile conditions. Then, GPR inversions of the field data are performed with the three models, the two-layered and profile inversions being limited to some parts of the field where specific profile conditions were observed. The surface soil moisture map from the one-layered inversion is presented and interpreted in the light of insitu observations. Finally, soil moisture maps from twolayered and profile inversions are compared, as well as soil moisture profiles.

\section{GPR forward and inverse mod- eling}

\subsection{GPR system modeling}

The GPR method is based on the propagation of an electromagnetic wave into the ground, which is governed by its electromagnetic parameters, i.e., the dielectric permittivity $\varepsilon$, the electrical conductivity $\sigma$ and the mag- 
netic permeability $\mu$. As the dielectric permittivity of water $\left(\varepsilon_{w} \approx 80\right)$ is much larger than the one of the soil particles $\left(\varepsilon_{s} \approx 5\right)$ and air $\left(\varepsilon_{a}=1\right)$, the GPR wave propagation in the soil is principally determined by its water content.

Following Lambot et al. [2004b, 2006], the GPR system is set up with a vector network analyzer (VNA) connected to an ultra wideband monostatic horn antenna situated off the ground. The VNA emulates a stepped-frequency continuous wave radar, that is, the GPR measurements are performed in the frequency domain. For this configuration, all antenna effects can be filtered out using the following equation where the GPR antenna is modeled as a linear system [Lambot et al., 2004b]:

$$
S_{11}(\omega)=H_{i}(\omega)+\frac{H(\omega) G_{x x}^{\uparrow}(\omega)}{1-H_{f}(\omega) G_{x x}^{\uparrow}(\omega)}
$$

where $S_{11}(\omega)$ is the quantity measured by the VNA, $H_{i}(\omega)$ is the antenna return loss, $H(\omega)$ is the antenna transmitting-receiving transfer function, $H_{f}(\omega)$ is the antenna feedback loss, $G_{x x}^{\uparrow}(\omega)$ is the transfer function of the air-subsurface system, the so-called Green's function, and $\omega$ is the angular frequency. A specific calibration of the radar antenna permits to determine the three frequency-dependent transfer functions $H_{i}(\omega)$, $H(\omega), H_{f}(\omega)$ and thus to obtain the $G_{x x}^{\uparrow}(\omega)$ from the raw measurements $S_{11}(\omega)$.

The electromagnetic model calculating the Green's function simulates the response of the soil illuminated by the GPR antenna, depending of the soil electromagnetic properties. It represents an exact solution of the 3D Maxwell's equations for electromagnetic wave propagation in a multilayered medium. The consideration of a 3-D model is essential to take into account spherical divergence (geometric spreading) in wave propagation. The soil can be discretized in multiple layers with homogeneous electromagnetic properties, i.e., the dielectric permittivity $\varepsilon$, the electrical conductivity $\sigma$ and the thickness of each layer $h$. A continuously variable medium can be modeled using layer thicknesses that are smaller than one tenth the wavelength. The reader is referred to Lambot et al. [2004b, 2006] for additional details on this model.

\subsection{Petrophysical relationships}

In this study, the petrophysical relationships between the soil moisture and its electromagnetic properties are described, respectively, by (1) the model of Ledieu et al. [1986] to derive the volumetric soil moisture $\theta$ from the relative dielectric permittivity $\varepsilon_{r}$ :

$$
\theta=a \sqrt{\varepsilon_{r}}+b
$$

with $a=0.1264$ and $b=-0.1933$ for a specific soil, and by (2) the model of Rhoades et al. [1976] to relate the soil electrical conductivity $\sigma$ to the soil moisture:

$$
\sigma=\left(c \theta^{2}+d \theta\right) \sigma_{w}+\sigma_{s}
$$

where the parameters are set to $c=1.85, d=3.85 \times$ $10^{-2}, \sigma_{w}=0.075 \mathrm{Sm}^{-1}$ and $\sigma_{s}=5.89 \times 10^{-4} \mathrm{Sm}^{-1}$. These parameters were determined in the laboratory for a specific soil subject to different water contents and salinities. Both dielectric permittivity and electrical conductivity are thus related to the soil moisture by these specific relationships throughout all the study.

\subsection{Inversion of GPR data}

The parameters of the multilayered medium investigated by the GPR are retrieved by a full-waveform inversion of the filtered radar signal, i.e., the Green's function. This inversion is done in the frequency domain, where the wave is actually modeled. The inverse problem is formulated in the least-squares sense and the objective function is accordingly defined as follows:

$$
\phi(\mathbf{b})=\left(\mathbf{G}_{\mathbf{x x}}^{\uparrow *}-\mathbf{G}_{\mathbf{x x}}^{\uparrow}\right)^{T}\left(\mathbf{G}_{\mathbf{x x}}^{\uparrow *}-\mathbf{G}_{\mathbf{x x}}^{\uparrow}\right)
$$

where $\mathbf{G}_{\mathbf{x x}}^{\uparrow *}$ and $\mathbf{G}_{\mathbf{x x}}^{\uparrow}$ are, respectively, the measured and the modeled Green's functions and $\boldsymbol{b}$ is the vector of parameters to be estimated. The model inversion thus consists of finding the minimum of this objective function. Optimization is performed using a local search algorithm (i.e., the Levenberg-Marquardt (L-M) algorithm) for the simplest models with few parameters to invert for and a combination of global and local optimization (i.e., the Global Multilevel Coordinate Search (GMCS) and the Nelder-Mead Simplex (NMS) algorithms) for the more complex models. In this study, the electrical conductivity of the soil is not optimized but is directly derived from the dielectric permittivity using (2) and (3).

\subsection{Multilayered medium models}

In this study, the soil is modeled using three types of multilayered media. Inversion of the GPR signals using the three different types of model permits to get 


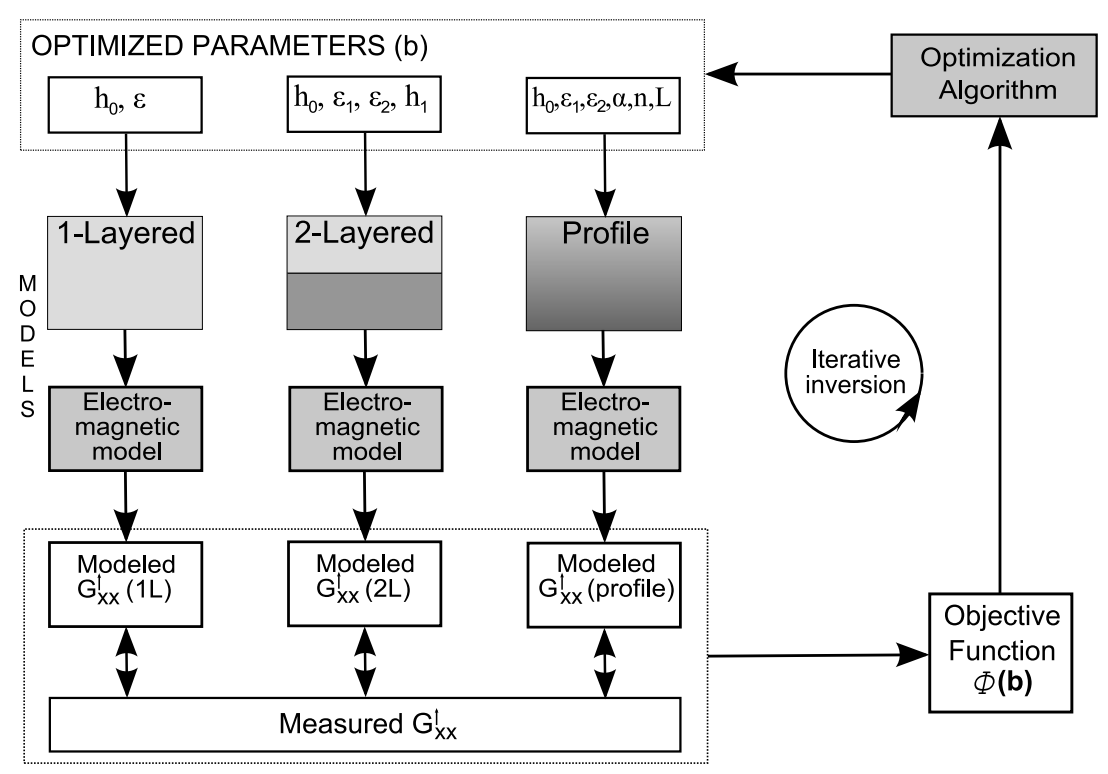

Figure 1: Flowchart of the inversion of the Green's function $\mathbf{G}_{\mathbf{x x}}^{\uparrow}$ with the three different multilayered soil moisture models, that are, the one-layered, two-layered and profile models.

different soil moisture descriptions. Fig. 1 shows the inversion flowchart for the three different multilayered models.

\subsubsection{One-layered model}

The first model, that is, the one-layered model, is the most simplified one as it assumes a homogeneous halfspace for the soil, below the air layer. Only two parameters are considered in the inversion, i.e., the soil dielectric permittivity $\varepsilon$ and the antenna height above the soil $h_{0}$, as the electromagnetic properties of the air layer are known $\left(\varepsilon_{a}=1\right.$ and $\left.\sigma_{a}=0 \mathrm{~S} / \mathrm{m}\right)$. This robust inversion procedure was used for soil moisture retrieval from GPR data in field conditions in [Weihermüller et al., 2007, Lambot et al., 2008b].

\subsubsection{Two-layered model}

The second model, that is, the two-layered model, assumes a two-layered soil with a top soil layer ( $1^{\text {st }}$ layer) above an half-space medium ( $2^{\text {nd }}$ layer), in addition to the air layer. This model permits to assess two different homogeneous soil dielectric permittivity values $\varepsilon_{1}$ and $\varepsilon_{2}$, the thickness of the top layer $h_{1}$ and the antenna height $h_{0}$. For small top layer thicknesses, constructive and destructive interferences in the GPR signal may occur, resulting in a less sensitivity of the model to its parameters. The method was validated in laboratory conditions against real measurements for these critical cases in [Lambot et al., 2006, Minet et al., 2010].

\subsubsection{Profile model}

The third model, that is, the profile model, assumes a soil where the soil moisture can vary continuously with depth, according to a hydrostatically coherent soil moisture profile. We chose to simulate soil moisture profiles with the model of van Genuchten [1980], which describes the water retention curve between two extreme soil moisture values at the top and the bottom of the profile. The model of van Genuchten giving the water retention curve as a function of the depth $z$ taken positively downwards is given below:

$\theta(z)= \begin{cases}\theta_{1}+\left(\theta_{2}-\theta_{1}\right)\left(1+|\alpha(L-z)|^{n}\right)^{-m} & \text { for } z<L \\ \theta_{2} & \text { for } z \geq L\end{cases}$

(5)

where $\theta$ is the volumetric soil moisture, $\theta_{1}$ and $\theta_{2}$ are the top and bottom soil moisture respectively, $L$ is the depth in which the profile is developed, $\alpha$ and $n$ are curve shape parameters, and $m=1-1 / n$ is restricted by Mualem's condition with $n>1$.

The soil moisture profiles must be transformed to dielectric permittivity profiles for the need of the elec- 
tromagnetic model. Introducing (2) into (5) leads to the equation describing the soil dielectric profiles as a function of the depth $z$ [Lambot et al., 2004a]:

$\varepsilon(z)=\left\{\begin{array}{l}{\left[\sqrt{\varepsilon_{1}}+\left(\sqrt{\varepsilon_{2}}-\sqrt{\varepsilon_{1}}\right)\left(1+[\alpha(L-z)]^{n}\right)^{-m}\right]^{2}} \\ \varepsilon_{2}\end{array}\right.$

where $\varepsilon_{1}$ and $\varepsilon_{2}$ are, respectively, the dielectric permittivity of the soil surface and of the soil at the depth $z=L$.

The dielectric profiles are discretized into $1 \mathrm{~mm}$ thick soil layers, resulting in maximum 250 layers in the electromagnetic model, as the depth of the profile $L$ is bounded to $0.25 \mathrm{~m}$ in the optimization. This high resolution permits to emulate the continuity of the profile for the computation of the Green's function as the layer thickness $(1 \mathrm{~mm})$ is far below the wavelength of the GPR signal. It is worth noting that the coupling of the van Genuchten's model to the electromagnetic model considerably decreases the number of unknowns in the optimization (from maximum $2 \times 250$ to 6 unknowns), the GPR inversion being constrained by the van Genuchten's model. The optimized parameters in the inversion are the van Genuchten parameters $\alpha, n$, $\theta_{1}, \theta_{2}$ and $L$ and the antenna height $h_{0}$. This approach was validated with numerical and laboratory experiments in Lambot et al. [2004a].

\section{Numerical experiments}

\subsection{Model configurations}

The numerical experiments aim to reproduce the real medium that was investigated with the GPR in field conditions and that is expected to be a continuous (and hydrostatically coherent to some extent in this case) soil moisture profile. Synthetic Green's functions were generated using the profile model, using dielectric profiles that were computed by the van Genuchten model. Different configurations of soil dielectric profiles were investigated, with $\alpha$ ranging from $16 \mathrm{~m}^{-1}$ to $30 \mathrm{~m}^{-1}$ and $n$ from 6 to 20 by a step of 2, resulting in total in 120 configurations. The other parameters of Equation (6) were fixed at $\varepsilon_{1}=3.12$ and $\varepsilon_{2}=12.30$, corresponding to soil moisture values of $\theta_{1}=0.03$ and $\theta_{2}=0.25$, respectively, and $L=0.10 \mathrm{~m}$. The profile was discretized in 100 soil layers of $1 \mathrm{~mm}$ thickness for generating the Green's functions using the multilayered electromagnetic model. Green's functions were computed in a wide frequency bandwidth, from 200 to $2000 \mathrm{MHz}$, with a frequency step of $6 \mathrm{MHz}$.

Then, synthetic Green's functions generated with the profile model were inverted with the two-layered model wfirhin thelsame frequency range, considering the optinfization of the first $\left(\varepsilon_{1}\right)$ and second layer $\left(\varepsilon_{2}\right)$ dielectric permittivities and of the first layer thickness $\left(h_{1}\right)$. The objective of the numerical experiments is to assess the reliability of the simplified two-layered model facing a continuous dielectric profile. Using a simplified model in practice is beneficial in terms of computing ressources that are needed. Indeed, the calculation time of the Green's function is almost proportional to the number of layers in the model.

\subsection{Numerical results}

\subsubsection{Soil moisture profile retrieval with the two- layered inversion}

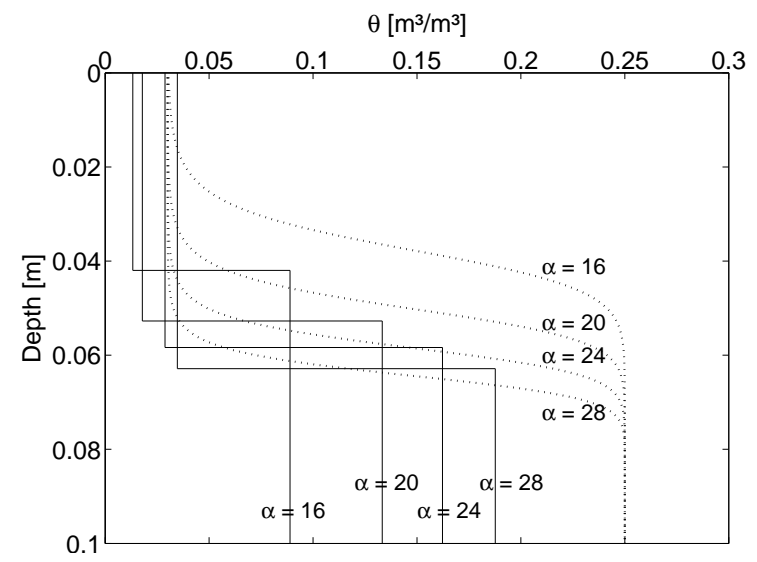

Figure 2: Numerically generated soil moisture profiles with the van Genuchten model (dotted lines) and twolayered profile (plain lines) retrieved by inversion using the two-layered model, for four different van Genuchten parametrizations ( $n=14$ and $\alpha=16,20,24$ and 28 $\left.\mathrm{m}^{-1}\right)$.

Fig. 2 shows the soil moisture profiles used for generating the Green's functions and the two-layered discontinuous profiles retrieved by two-layered model inversions, for four different configurations. The parameters of the model of van Genuchten for the configurations shown here are $n=14$ and $\alpha=16,20,24$ and $28 \mathrm{~m}^{-1}$, respectively. The depths of the interfaces between the two layers in the two-layered profiles are set 
to the first layer thicknesses $h_{1}$ retrieved by inversion. It is worth mentioning that inversions performed with the same profile model as the one used for generating the synthetic Green's functions led to exactly the same parameters values that the values used for generating the data.

The general trend of the profile, that is, the soil moisture increases with depth, is always respected in the two-layered profile, i.e., $\theta_{1}<\theta_{2}$. This relation can be observed for all the 120 configurations (not shown). The first layer soil moisture values retrieved by twolayered inversions $\theta_{1}$ are very close to the top soil moisture from the profile model. However, the second layer soil moisture values $\theta_{2}$ are underestimated compared to the soil moisture at the bottom of the profile, especially for configurations where $\alpha=16$ and $20 \mathrm{~m}^{-1}$. This poorer performance of the two-layered inversion in the retrieval of the second layer soil moisture compared to the first layer soil moisture is to be attributed to the lower information content in the GPR waves reflection from deeper soil layers. Although there is a larger dielectric contrast between the two extreme soil permittivity values $\left(\Delta \varepsilon_{1,2}=9.18\right)$ than between the air and the soil surface values $\left(\Delta \varepsilon_{a i r, 1}=2.12\right)$, the smooth continuously-varying interface between the two extreme soil permittivities leads to a less well-defined wave reflection than the clear-cut interface between the air and soil surface. Hence, for configurations where the profile is more abrupt (e.g., $\alpha=28 \mathrm{~m}^{-1}$ ), the retrieved second layer soil moisture is better matching the bottom soil moisture of the continuous profile. In addition, GPR waves are attenuated while penetrating deeper into the soil because of the electrical losses due to the nonnull electrical conductivity. Even though this is accounted for in the electromagnetic model, it reduces the information content in the soil subsurface waves reflections. These two reasons result in a less sensitivity of the two-layered model to the parameters of the second layer. A better confidence for the first layer soil moisture compared to the second layer soil moisture can then be expected when using a two-layered model facing a continuously-varying soil moisture profile, especially for smooth profiles.

\subsubsection{Comparison of interfaces depth between two- layered and profile inversions}

For the four configurations shown in Fig. 2, the inverted first layer thickness $h_{1}$ retrieved by the two-layered inversion seems to match the depth at which the gener-

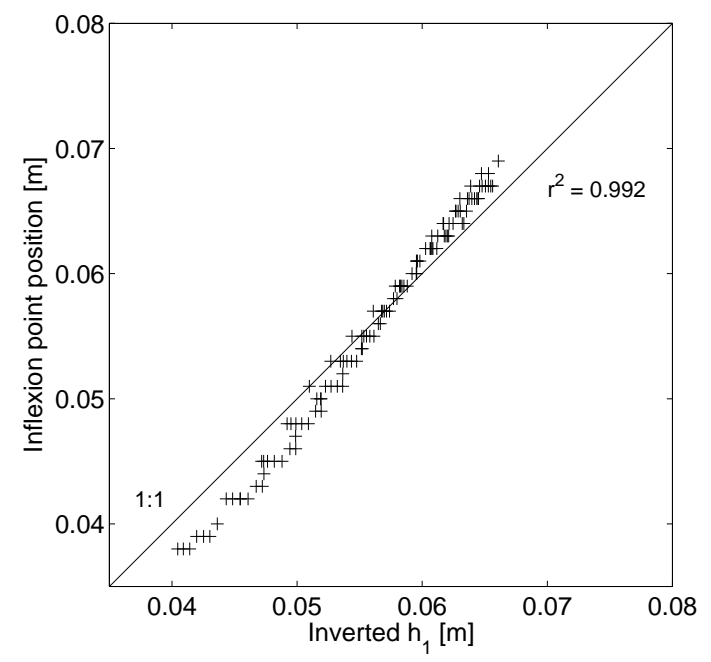

Figure 3: Comparison between the first layer thickness $h_{1}$ retrieved by inversion using the two-layered model and the position of the inflexion point $z_{i}$ of the generated soil moisture profile.

ated soil moisture profile is abruptly increasing (inflexion point). Fig. 3 compares, for all the configurations, the first layer thickness $h_{1}$, that is, the depth of the interface between the two layers, and the position of the inflexion point of the van Genuchten soil moisture profiles $z_{i}$, determined by solving $\frac{\delta^{2} \theta\left(z_{i}\right)}{\delta z^{2}}=0$. There is a very good agreement between these two variables, with a coefficient of correlation of $r^{2}=0.992$. Maximum difference between the two variables is about 4 $\mathrm{mm}$, which is very accurate knowing that the numerical resolution of the profile (i.e., the size of the layers) is $1 \mathrm{~mm}$ and that the traditional GPR resolution (one fourth the wavelength) is equal to $0.021 \mathrm{~m}$ for a dielectric permittivity of 3.2 and for the highest frequency (2000 MHz). The use of a two-layered model to reconstruct continuously-varying profiles shows therefore a great potential to retrieve the depth of the interface between two extreme soil moistures, as well as the surface soil moisture. 


\section{GPR Field Measurements}

\subsection{Materials and methods}

\subsubsection{Study site and weather conditions}

The 11-ha cultivated field that was mapped with the GPR is located in the loess belt area near the locality of Walhain in the center of Belgium (Long. $4^{\circ} 41^{\prime} 8^{\prime \prime} \mathrm{E}$, Lat. $\left.50^{\circ} 35^{\prime} 59^{\prime} \mathrm{N}\right)$. Soil type is uniformly a silt loam, with a gentle topography ranging from 153 to $161 \mathrm{~m}$ a.s.l and slope lower than $7 \%$. The campaign took place at the end of the winter on the $23^{\text {th }}$ of March 2009, while the field was covered by winter barley with a canopy height less than five $\mathrm{cm}$. Soil was relatively dry as no rainfall were recorded for eight days according to a weather station located at around ten $\mathrm{km}$ from the field ${ }^{1}$.

The soil moisture was suspected to increase with depth, as we observed in-situ a dry superficial soil layer above a wetter subsurface soil. An abrupt profile or two-layered conditions were thus supposed to be encountered. Soil moisture volumetric sampling could not be performed to assess actual soil layering as the dry superficial layer was very thin $(<5 \mathrm{~cm})$. In addition, comparison of GPR-derived soil moisture with volumetric ground sampling measurements is expected to be cumbersome and suspicious due to the inherent high spatial variability of soil moisture both vertically and horizontally, to the different support scales of the measurement techniques and to the uncertain petrophysical relationship that is used to convert the dielectric permittivity into volumetric water content. Due to soil properties variability (e.g., density), the petrophysical relationship is thus expected to vary drastically across the field. Therefore, we decided not to take soil samples for this study.

\subsubsection{GPR platform}

The GPR system was set up by combining a vector network analyzer (ZVL, Rohde \& Schwarz, Münich, Germany) with a linear polarized double-ridged broadband horn antenna (BBHA 9120 F, Schwarzbeck MessElektronik, Schönau, Germany). Antenna dimensions are $950 \mathrm{~cm}$ in length and $680 \times 960 \mathrm{~cm}^{2}$ in aperture area and the nominal frequency range is from 0.2 to $2 \mathrm{GHz}$. The antenna was connected to the reflection port of the VNA with a high quality $\mathrm{N}$ type $50-\Omega$ coax-

\footnotetext{
${ }^{1}$ ASTR-UCL

Weather http://www.astr.ucl.ac.be/data/meteo-lln/live/index.html
}

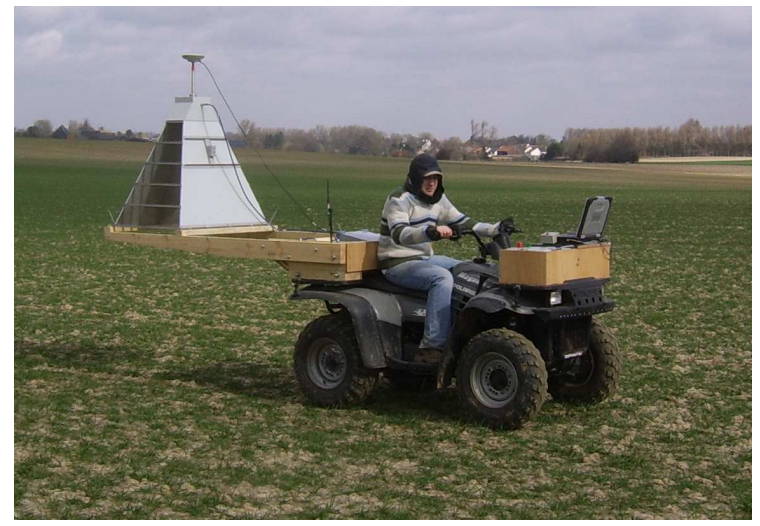

Figure 4: Four-wheel motorcycle holding the GPR system constituted of a horn antenna linked to a vector network analyzer, the DGPS device and the laptop. Picture taken on the $23^{\text {th }}$ of March 2009 in a barley field near Walhain, Belgium.

ial cable of $2.5 \mathrm{~m}$ length. We calibrated the VNA at the connection point at the end of the cable before the antenna feed point using a $50-\Omega$ OSM (Open, Short, Match) calibration kit (ZVZ21-N, Rohde \& Schwarz). The frequency-dependent complex ratio $S_{11}$ between the returned and the emitted signal was measured sequentially at 301 stepped frequencies from 200 to 2000 $\mathrm{MHz}$, with a frequency step of $6 \mathrm{MHz}$.

The GPR system was mounted on a four-wheel motorcycle to allow for fast data acquisition. Fig. 4 shows a picture of the motorized platform that we used for acquiring the data. The GPR was automatically controlled by a PC and combined with an accurate differential global positioning system device (DGPS) (Leica GPS1200, Leica Geosystems) for the georeferencing of the measured points, with a precision of about $3 \mathrm{~cm}$. Real-time GPR measurements were performed at a regular distance spacing of two meters in the same track, according to the DGPS position. The distance spacing between the tracks was about ten meters. In total 3741 GPR measurements were taken across the field in $3 \mathrm{~h} 48$, with a driving speed of about $5 \mathrm{~km} / \mathrm{h}$.

\subsubsection{GPR field data inversions}

The raw measured signals $S_{11}(\omega)$ were first filtered from the antenna effects using (1) to obtain the Green's functions $G_{x x}^{\uparrow}(\omega)$ from the soil. Inversions of the $G_{x x}^{\uparrow}(\omega)$ were conducted with the three different models described above, namely, the one-layered, the two- 
Table 1: Summary of the inversions of GPR field data for the three models.

\begin{tabular}{|c|c|c|c|c|c|c|c|}
\cline { 2 - 8 } \multicolumn{1}{c|}{} & $\begin{array}{c}\text { Number of } \\
\text { points }\end{array}$ & $\begin{array}{c}\text { Number of } \\
\text { parameters }\end{array}$ & $\begin{array}{c}\text { Number of } \\
\text { soil layers }\end{array}$ & $\begin{array}{c}\text { Frequency } \\
\text { range [MHz] }\end{array}$ & $\begin{array}{c}\text { Optimization } \\
\text { algorithms }\end{array}$ & $\begin{array}{c}\text { Number of } \\
\text { iterations }\end{array}$ & $\begin{array}{c}\text { CPU } \\
\text { time }^{a}\end{array}$ \\
\hline 1-layered & 3741 & 2 & 1 & $200-800$ & L-M & $\sim 20$ & 1 \\
2-layered & 300 & 4 & 2 & $200-1600$ & GMCS-NMS & 6400 & 170 \\
Profile & 300 & 6 & $1-250$ & $200-1600$ & GMCS-NMS & 14400 & 12000 \\
\hline
\end{tabular}

${ }^{a} \mathrm{CPU}$ time is the relative time of computation for one GPR inversion compared to the one-layered inversion.

layered and the profile models. For the simplified onelayered model, GPR data were selected within the frequency range from 200 to $800 \mathrm{MHz}$, as noise in the signal appears at frequencies higher than $800 \mathrm{MHz}$. Noise arises at high frequencies due to interferences caused by the soil roughness and the presence of vegetation. For roughness approaching one quarter the GPR wavelength, constructive and destructive interferences may affect the GPR signal. Therefore, rejection of high frequencies (i.e., small wavelengths) permits to avoid interference problems, although it results in a poorer wave resolution for detecting small layers.

For the two-layered and the profile models, a more extended frequency range from 200 to $1600 \mathrm{MHz}$ is necessary to get more information about the layered or profile structure. According to in-situ observations, we selected 300 points where an abrupt soil moisture profile was expected to be encountered. Only these selected points were thus inverted considering twolayered and profile models. Inversions were set in a large parameter space, namely, $0 \leq \varepsilon_{1} \leq 25,0 \leq \varepsilon_{2} \leq$ $25,1.45 \leq h_{0} \leq 1.75 \mathrm{~m}$ and $0 \leq h_{1} \leq 0.25 \mathrm{~m}$ for the two-layered inversions and $1 \leq \alpha \leq 75 \mathrm{~m}^{-1}, 1 \leq n \leq$ $75,0 \leq \varepsilon_{1} \leq 12,0 \leq \varepsilon_{2} \leq 25,1.45 \leq h_{0} \leq 1.75 \mathrm{~m}$ and $0 \leq L \leq 0.25 \mathrm{~m}$ for the profile inversions. Table 1 summarizes the status of the three inversions performed on the GPR field data.

\subsection{Field measurements results}

\subsubsection{Map of surface soil moisture from the one- layered model inversion}

Fig. 5 shows the soil moisture point-data retrieved by one-layered model inversions of the 3741 points that were measured during the GPR field acquisition campaign. Inverted dielectric permittivities were translated into soil moisture values using (2). Size of the symbols are varying according to a weight function based on the root mean square error (RMSE) between the amplitude of measured and modeled (inverted) Green's functions

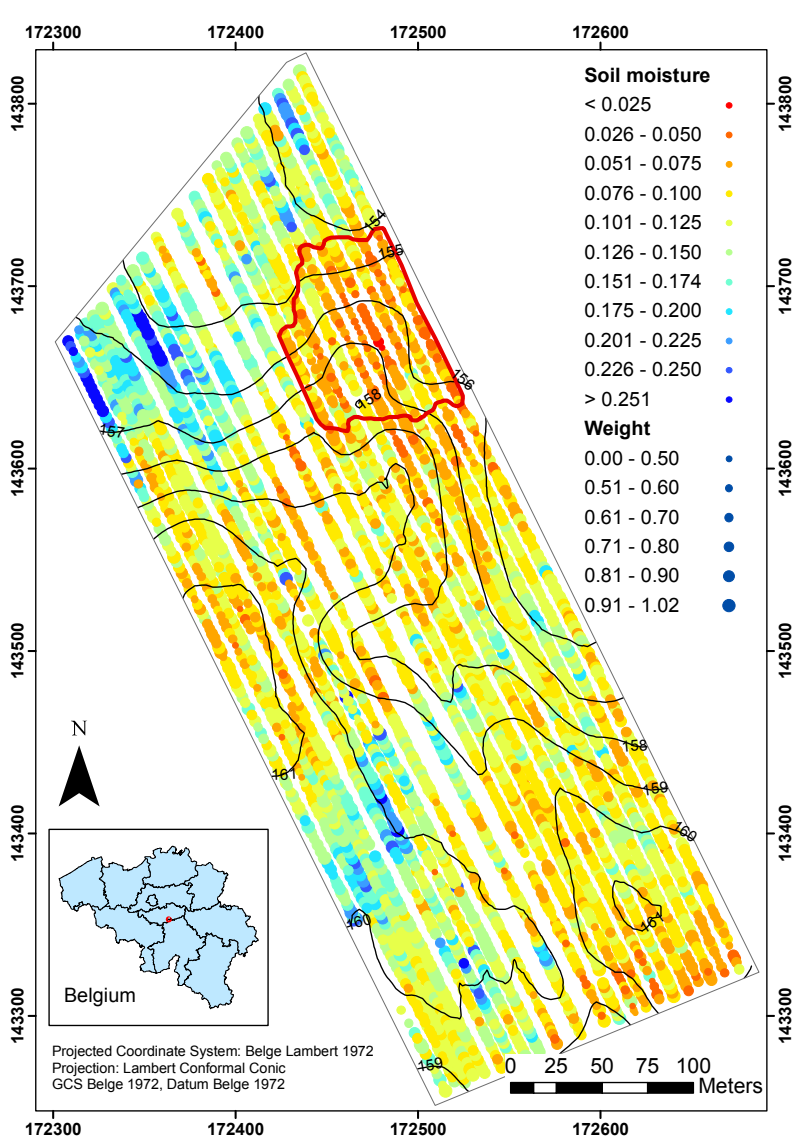

Figure 5: Soil moisture map retrieved by one-layered model inversions from the field acquisition near Walhain on the $23^{\text {th }}$ of March 2009. Size of the symbols are inversely related to their uncertainty by the weight function, which is, Weight $=\max (R M S E)-R M S E$. 
$($ Weight $=\max (R M S E)-R M S E)$. The RMSE is an indicator of the uncertainty associated with the inversion of the measured signal. Topography is depicted with black contour lines with a contour interval of $1 \mathrm{~m}$. The red polygon located in the driest part of the map delineates the 300 manually selected points for the twolayered and profile inversions.

The mean volumetric soil moisture is equal to 0.108 and its standard deviation is 0.0401 . These values are similar to the ones observed in previous studies for the same extent and similar support depth (e.g., [Famiglietti et al., 2008], just to cite one). It is worth mentioning that the 300 selected points for the two-layered and profile inversions are affected by a large uncertainty (i.e., smaller points on the map), due to their large RMSE between measured and inverted signals. These large RMSE values indicate that one-layered model fails to adequately reproduce the Green's functions at these points because of the non-uniform moisture conditions.

At the global pattern scale, soil moisture is mainly explained by topography. A wet area can be observed in the North of the field, where a thalweg flows in the North-North-East direction. Another wet area can be observed in the South-West part of the field, which is characterized by a flat area. Hilltops are mainly dry, like in the South-East corner and around the zone delineated by the red polygon.

A close examination of the map reveals that the spatial local pattern of soil moisture exhibits a clear line effect. This line effect is translated by a high spatial continuity for data retrieved along the sampling line, whereas at the same time there is an abrupt change in soil moisture when moving to adjacent lines. This effect is clearly visible e.g., on the top left corner of Fig. 5 , where there are adjacent segments of high or low water contents.

Investigating the variogram by separating its estimates along the lines and perpendicular to the lines clearly confirms this effect (Fig. 6). The variogram estimated along the lines shows smoothly increasing variance with distance, with a moderate nugget effect accounting for about $25 \%$ of the sampling variance, thus emphasizing the rather progressive changes of water content, with correlated values up to about 130 meters. In contrast, the variogram perpendicular to the lines is offset by a value that corresponds to the additional nugget effect induced by the between-lines variability, which tends to mask the global pattern of volumetric soil moisture. It can presumably be assumed that the line effect is the result of the dragging line laid by

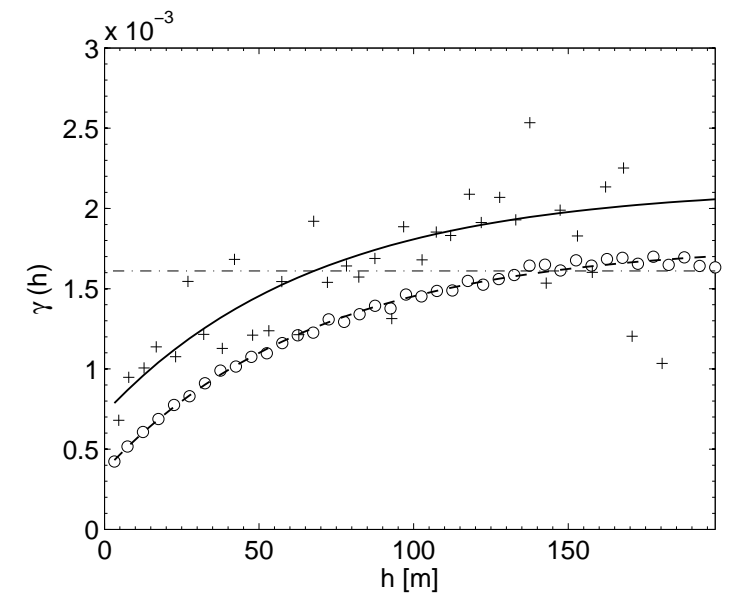

Figure 6: Variogram for soil moisture computed on the whole field with a class distance from 0 to $200 \mathrm{~m}$ by a step of $5 \mathrm{~m}$. Variogram along and perpendicular to the sampling line is denoted by circle and plus symbols, respectively. Exponential models are fitted for both variograms. The sampling variance is indicated by horizontal dashed line.

ploughing or fertilizer spreading machine, as the machine causes compaction layer along the track of the wheels.

Though these findings are not completely validated yet, further application such as mapping continuous soil moisture content could require some subsequent work in order to remove the line effect and improves the estimation and mapping of the moisture content in the field.

\subsubsection{Maps of surface and subsurface soil moisture from two-layered and profile inversions}

Fig. 7 shows the first layer (a) and second layer (b) soil moisture maps from two-layered inversions and the top (c) and bottom (d) soil moisture maps from profile inversions, for the 300 selected points. Soil moisture values are depicted with the same color range from 0 to 0.25 in the four maps.

Considering two-layered model inversions, first layer soil moisture values (Fig. 7(a)) are very low, indicating a completely dry soil at the wilting point, as it was visually observed in the field. Second layer soil moisture map (Fig. 7(b)) shows globally higher values and a less spatially coherent structure (more speckle noise). This noise could originate from the lower sensitivity of the two-layered model to the second layer dielectric 


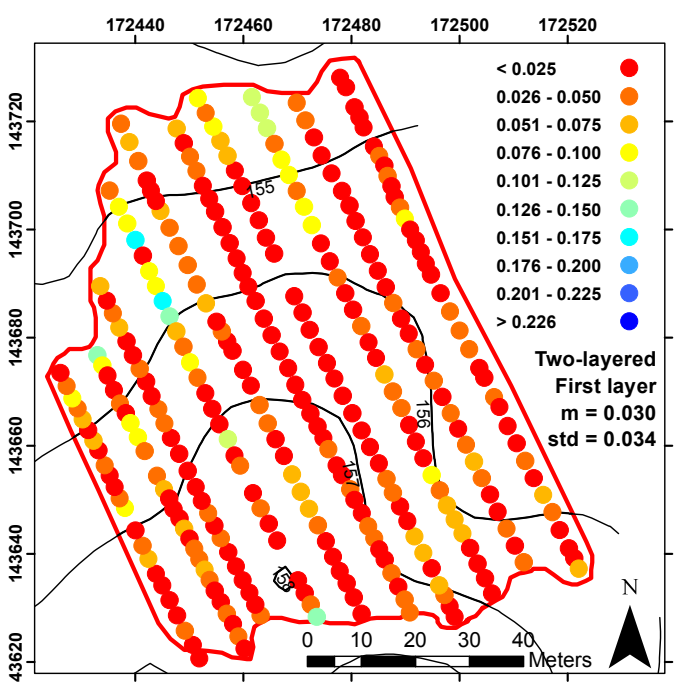

(a)

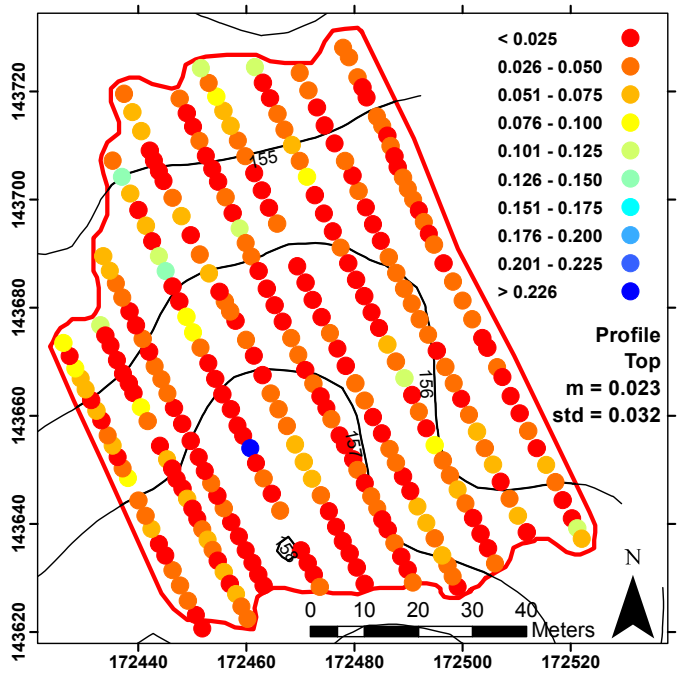

(c)

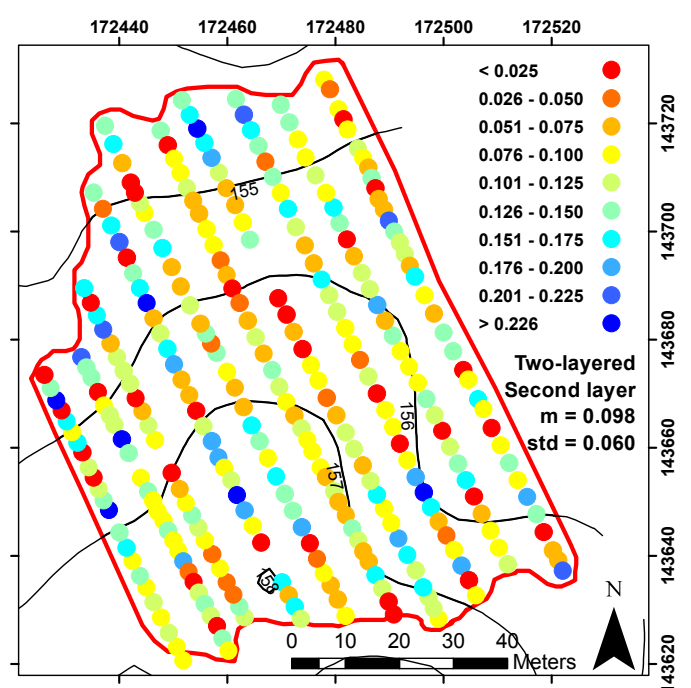

(b)

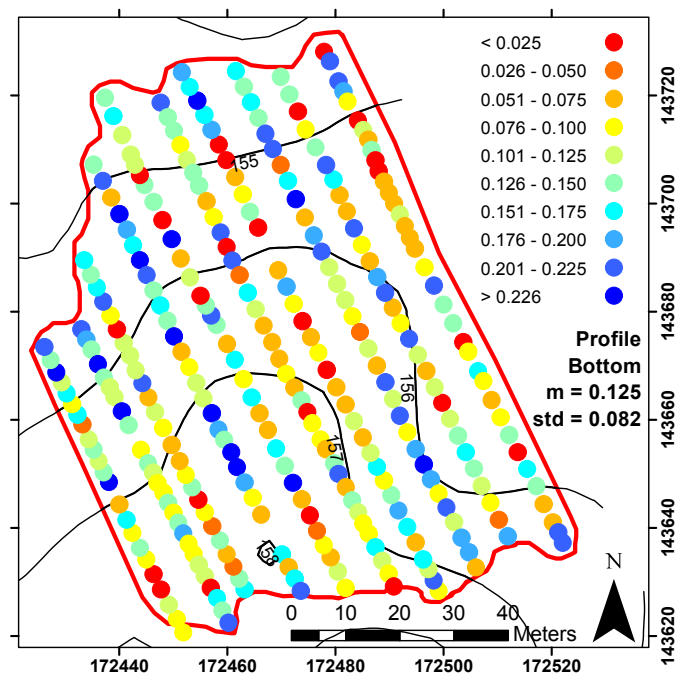

(d)

Figure 7: Soil moisture maps from two-layered model inversions for the first layer (a) and the second layer (b) and from the profile model inversions for the top (c) and the bottom (d) of the profile. Mean $(m)$ and standard deviation $(s t d)$ of soil moisture are depicted below the legend in each map. 
permittivity, as it was already explained in the numerical experiments. As a result, second layer soil moisture values are more subject to uncertainties than the first layer soil moisture. First layer soil moisture values $\theta_{1}(2 L)$ are smaller than second layer soil moisture values $\theta_{2}(2 L)$ in $265(88.3 \%)$ points, that is, at these locations, soil moisture increases with depth, as it was expected from in situ observations. Moreover, comparing with one-layered model inversions, the condition $\theta_{1}(2 L)<\theta(1 L)<\theta_{2}(2 L)$, where $\theta(1 L)$ is the value retrieved by the one-layered inversion, is respected in $204(68.0 \%)$ points. The optimized first layer thickness (not shown) varies from 2 to $20 \mathrm{~cm}$, although most of the values are around $3 \mathrm{~cm}(71.3 \%$ of the points show first layer thickness values between 2 and $4 \mathrm{~cm}$ ).

Soil moisture maps from the profile inversions show similar patterns than the maps from the two-layered inversions, particularly when comparing first layer values (Fig. 7(a)) and soil moisture values at the top of the profile (Fig. 7(c)). Soil moisture values at the bottom of the profile (Fig. 7(d)) seem higher than the second layer values (Fig. 7(b)) in the two-layered inversions, probably because of the deeper characterization of the soil moisture with the profile inversion. Top soil moisture values $\theta_{1}$ (profile) are smaller than bottom soil moisture values $\theta_{2}$ (profile) in $280(93.3 \%)$ points, while the condition $\theta_{1}$ (profile) $<\theta(1 L)<\theta_{2}$ (profile) is respected in $221(73.7 \%)$ points. The profile coherence is therefore more widely observed with the profile model than with the two-layered model inversions.

Comparing with the one-layered soil moisture map, surface soil moisture values (Fig. 7(a) and (b)) are closer to the one-layered soil moisture values (Fig. 5) than subsurface values, indicating that the one-layered model is restricted to the soil surface. One-layered inversions are therefore mainly sensitive to the surface reflection at the interface between soil and air.

\subsubsection{Comparison of soil moisture profile between inversion models}

Fig. 8 presents soil moisture profiles retrieved from the two-layered and the profile inversions and a unique soil moisture value retrieved from the one-layered inversion, for two GPR measured points. Both inversions with the two-layered and profile models at these two points show an increase of the soil moisture with depth. The top and bottom soil moisture values from the continuous profile inversions are very close, respectively, to the first and second layer soil moisture values from the two-layered inversions. One-layered soil moisture values are retrieved in the interval between the top and bottom soil moisture values of the profiles. For the point in Fig. 8(b), the continuous profile inversion leads to an abrupt soil moisture profile sharply following the discontinuous two-layered dielectric profile. It is worth mentioning that a majority of measured points shows a similar behavior, with soil moisture profiles that are identical between two-layered and profile inversions.

The abrupt soil moisture profiles retrieved by the profile inversion could originate from a lack of resolution of the GPR wave. Considering a dielectric permittivity of 5.5 (corresponding to the first layer soil moisture of 0.103 , the GPR resolution at the maximal frequency of $1600 \mathrm{MHz}$ is equal to $0.020 \mathrm{~m}$. Thus, inversions may be insensitive to continuous variations occurring within a very thin layer. This can also indicate real abrupt soil moisture profile conditions, resulting from a physical decoupling (strong pressure head gradient due to surface evaporation) of the soil water between the soil surface and subsurface.

\subsubsection{Comparison of interfaces depth retrieval be- tween two-layered and profile inversions}

For the two points depicted in Fig. 8, positions of the interfaces retrieved by the two-layered model inversions correspond quite well to the positions of the inflexion point of the continuous profile inversion, indicating that both models retrieve the same depth where the soil moisture is supposed to increase rapidly.

However, for all the 300 selected points, two-layered and profile inversions locate the interfaces at the same depth with a tolerance of $\pm 4 \mathrm{~mm}$ for 144 (48.0\%) points only, contrarily to the very good agreement that was observed in the numerical experiments with the same tolerance (Fig. 3). This poorer performance with some field data are to be attributed to the lowest maximum frequency of the GPR signal $(1600 \mathrm{MHz}$ instead of $2000 \mathrm{MHz}$ ), to the measurement noise arising with real data, and to model inadequacies with respect to the actual conditions that may not be adequately modeled with either a two-layered or a coherent soil moisture profile.

\subsubsection{Comparison between measured and modeled Green's functions}

Fig. 9 shows measured and modeled Green's functions $G_{x x}^{\uparrow}(\omega)$ with the one-layered model, the two-layered 


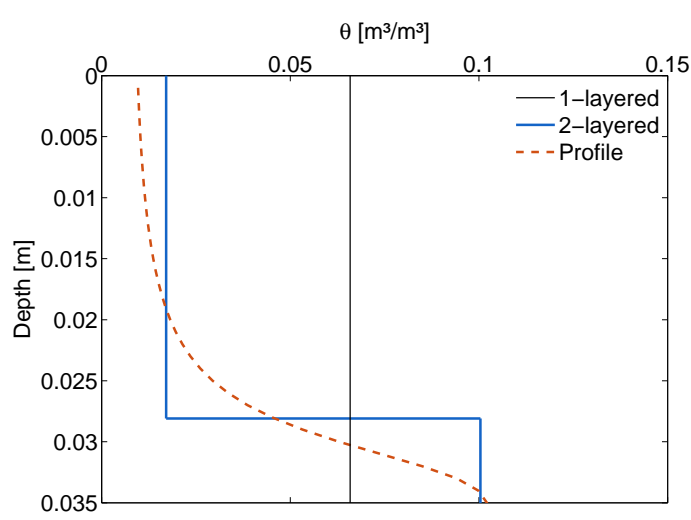

(a)

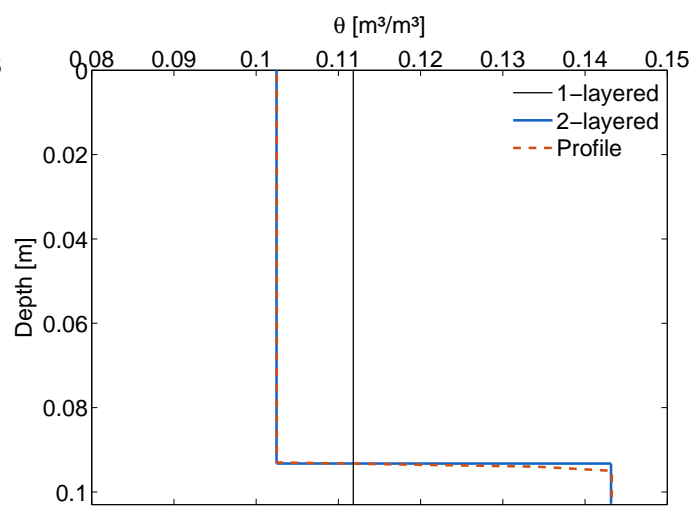

(b)

Figure 8: Soil moisture profiles retrieved from the two-layered inversion and the model inversion and unique soil moisture value retrieved from the one-layered model inversion for two GPR measured points ((a) and (b)).

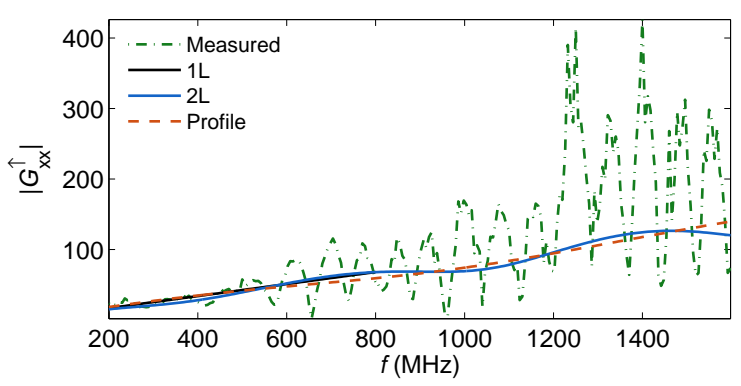

(a)

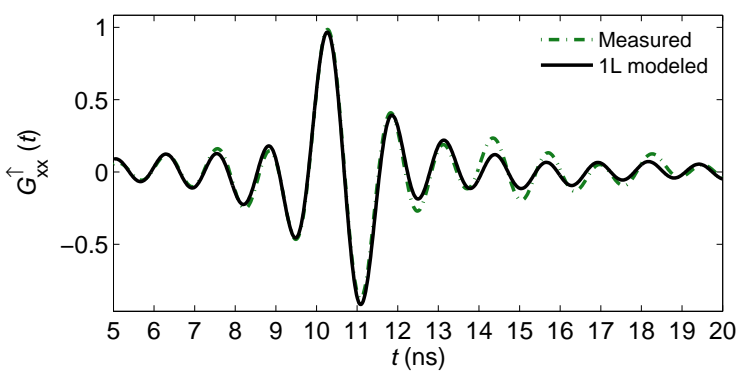

(c)

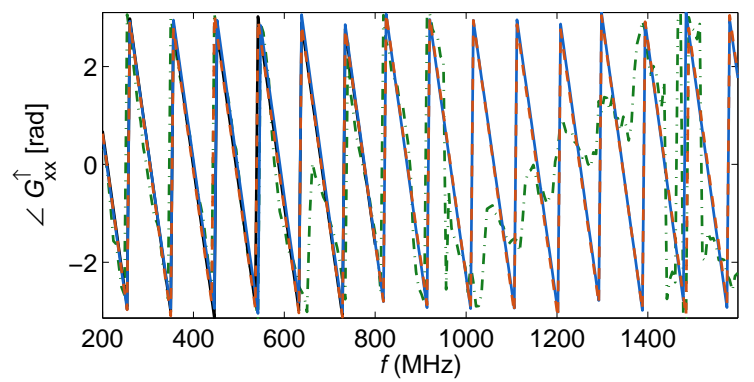

(b)

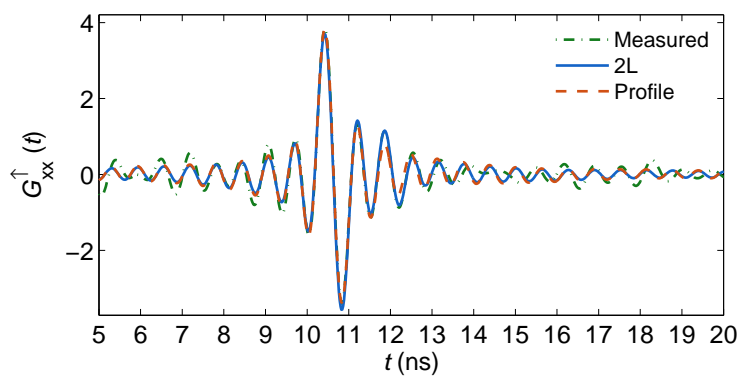

(d)

Figure 9: Measured and modeled Green's functions in the frequency domain depicted in amplitude (a) and phase (b) and in time domain for the one-layered model (c) and for the two others models (d). The one-layered modeled Green's function is defined on the frequency range from 200 to $800 \mathrm{MHz}$. Two-layered and profile modeled Green's functions are defined on the frequency range from 200 to $1600 \mathrm{MHz}$. 
model and the profile model, in frequency and time domains. The GPR measurement presented here corresponds to the point whose inverted profiles are depicted in Fig. 8 (b). The inversions for the one-layered model were performed on the limited frequency range 200$800 \mathrm{MHz}$, whereas the other inversions were performed in the frequency range 200-1600 MHz. Time domain representation of the Green's functions is achieved by applying an inverse Fourier transform to the frequency domain signal. As frequency bandwidths are different between the one-layered model and the two other models, the time domain representations must be separated.

In the frequency domain, discrepancies between measured and modeled signals appear very large, both in amplitude and in phase of the signal, especially at high frequencies above $1200 \mathrm{MHz}$. A regular oscillation of the measured signal with a small frequency period can be observed in the amplitude from $600 \mathrm{MHz}$, but this oscillation could not be modeled. This oscillation may correspond actually to a far-field reflector situated in the subsurface, that the two-layered or the profile models cannot reproduce because the maximum depth at which a reflector can be found for these models is bounded at $0.25 \mathrm{~m}$. As the noise appears to increase with frequency, i.e., with small wavelengths, this could also originate from surface soil roughness interferences, which produce scattering in the reflected radar waves when the wavelength is approaching the roughness height. Nevertheless, such discrepancies between measured and modeled signals are not observed in the time domain representation, both for one-layered (Fig. 9 (c)) and two-layered or profile models (Fig. 9 (d)). Even though the inversion is performed in the frequency domain, the good agreement between measured and modeled signals in the time domain indicates that the models used for simulating the Green's functions can adequately reproduce the GPR signal in the soil up to $0.25 \mathrm{~m}$ in depth. No differences could be observed between the two-layered and profile modeled signals, similarly to the soil moisture profile presented for this point in Fig. 8 (b). In the limited frequency range from 200 to $800 \mathrm{MHz}$, the two-layered and profile modeled signals also follow sharply the one-layered modeled signal.

As the first layer thickness that is to be retrieved is small, it corresponds in the frequency domain to a large oscillation, whose period is covering around $600 \mathrm{MHz}$ (e.g., local maxima of the amplitude in the frequency domain can be observed around 800 and $1400 \mathrm{MHz}$ for the two-layered modeled signal). As a result, a large frequency bandwidth (i.e., $1400 \mathrm{MHz}$ ) is required to model such small layers or profiles with two-layered and profile models. The use of these models on a limited frequency bandwidth as the one-layered model inversion would hamper the retrieval of the layering conditions.

In the time domain, the air-soil surface reflection peak is centered at $10.5 \mathrm{~ns}$ and is highly visible in both Fig. 9 (c) and (d). The signals oscillations are larger in Fig. 9 (c) than in Fig. 9 (d) because of the lowest frequencies (200 to $800 \mathrm{MHz}$ ) used in the one-layered inversion. Furthermore, the second reflection peak, i.e., between the two soil layers, can be detected around 12 ns. It remarkably well corresponds to the travel time in the first layer computed knowing the inverted parameters of the first layer with the two-layered model. For this point, the first layer thickness being around $0.1 \mathrm{~m}$ and the permittivity around 5 (see Fig. 8 (b)), the travel time in the first layer is equal to $1.5 \mathrm{~ns}$, which is corresponding to the position in time after the surface reflection peak.

\section{Conclusions and perspectives}

We analyzed the inversions of GPR data using three models, i.e., a one-layered, a two-layered and a continuously-variable profile model for soil moisture retrieval facing non-uniform conditions of soil moisture in depth. Numerical experiments showed that twolayered inversions permit to retrieve coherent soil moisture profiles, especially for the depth of the interface and for surface soil moisture, although more uncertainties were attributed to the second layer soil moisture.

A high-resolution GPR survey, including 3741 records over an 11-ha agricultural field, was conducted and data were analyzed using the three inversion strategies tested for the numerical experiments. One-layered inversions led to a consistent surface soil moisture map, with a global soil moisture pattern mainly explained by the topography whereas local patterns indicate a line effect presumably caused by the ploughing line. Surface and subsurface soil moisture maps from two-layered and profile inversions showed similar values and coherent soil moisture profile with respect with terrain observations, that is, soil moisture increases with depth. Retrieval of the same interface depth was more subject to discrepancies compared to numerical experiments, due to measurement noise and model inadequacy with real conditions.

Finally, comparing measured and modeled signals, 
inversion fits were very good in the time domain, while discrepancies were observed in frequency domain. These discrepancies were mainly attributed to measurement noise due to surface roughness and to the presence of reflectors outside of the $0-0.25 \mathrm{~m}$ soil depth. The full-waveform inversion of the radar signal inherently maximizes the information capability retrieval owing to the large frequency bandwidth (1400 $\mathrm{MHz}$ ), which is necessary to model shallow layering and soil moisture profile.

Further terrain evidences are needed to fully validate the technique, although the ground-truthing of soil moisture profiles remains complicated to perform at the field scale. Moreover, temporal dynamics of soil moisture patterns analysis, as well as the modeling of these patterns by the topography and soil type, would provide more insights about the reliability of the technique.

The off-ground GPR system presented in this study is a promising proximal soil sensing tool for highresolution and real-time soil moisture mapping at the field scale and for soil moisture profile retrieval, when profiles conditions are encountered. It could be used for the validation of microwave remote sensing products by ground-based surveys, improvement of hydrological modeling at the field or subcatchment scale and other environmental studies and applications.

\section{Acknowledgements}

We acknowledge Maxime Dupriez for the field assistance during the field campaign, as well as Guido Rentmeesters for the GPR platform construction. Easy access to the field was given by M Rieulaux, farmer at Walhain-St-Paul. The research presented in this paper was funded by the Belgian Science Policy Office in the frame of the Stereo II programme - project SR/00/100 (HYDRASENS), the DIGISOIL project financed by the European Commission under the 7th Framework Programme for Research and Technological Development, Area "Environment", Activity 6.3 "Environmental Technologies", and the Fonds de la Recherche Scientifique (FNRS), Belgium.

\section{References}

A. Binley, P. Winship, R. Middleton, M. Pokar, and J. West. High-resolution characterization of vadose zone dynamics using cross-borehole radar. Water Resources Research, 37(11):2639-2652, 2001.

W. J. Capehart and T. N. Carlson. Decoupling of surface and near-surface soil water content: A remote sensing perspective. Water Resources Research, 33 (6):1383-1395, 1997.

A. Ceballos, K. Scipal, W. Wagner, and J. MartinezFernandez. Validation of ERS scatterometer-derived soil moisture data in the central part of the Duero Basin, Spain. Hydrological Processes, 19(8):15491566, 2005.

J. A. Doolittle, B. Jenkinson, D. Hopkins, M. Ulmer, and W. Tuttle. Hydropedological investigations with ground-penetrating radar (GPR): Estimating watertable depths and local ground-water flow pattern in areas of coarse-textured soils. Geoderma, 131(3-4): 317-329, 2006.

G. D'Urso and M. Minacapilli. A semi-empirical approach for surface soil water content estimation from radar data without a-priori information on surface roughness. Journal of Hydrology, 321(1-4):297-310, 2006.

J. S. Famiglietti, D. Ryu, A. A. Berg, M. Rodell, and T. J. Jackson. Field observations of soil moisture variability across scales. Water Resources Research, 44:W01423, 2008.

L. W. Galagedara, G. W. Parkin, J. D. Redman, P., and A. L. Endres. Field studies of the GPR ground wave method for estimating soil water content during irrigation and drainage. Journal of Hydrology, 301: 182-197, 2005.

J. A. Huisman, S. S. Hubbard, J. D. Redman, and A. P. Annan. Measuring soil water content with ground penetrating radar: A review. Vadose Zone Journal, 2:476-491, 2003.

S. Lambot, J. Rhebergen, I. van den Bosch, E. C. Slob, and M. Vanclooster. Measuring the soil water content profile of a sandy soil with an off-ground monostatic ground penetrating radar. Vadose Zone Journal, 3(4): 1063-1071, 2004a.

S. Lambot, E. C. Slob, I. van den Bosch, B. Stockbroeckx, and M. Vanclooster. Modeling of groundpenetrating radar for accurate characterization of subsurface electric properties. IEEE Transactions 
on Geoscience and Remote Sensing, 42:2555-2568, 2004b.

S. Lambot, L. Weihermüller, J. A. Huisman, H. Vereecken, M. Vanclooster, and E. C. Slob. Analysis of air-launched ground-penetrating radar techniques to measure the soil surface water content. Water Resources Research, 42:W11403, 2006.

S. Lambot, A. Binley, E. C. Slob, and S. Hubbard. Ground penetrating radar in hydrogeophysics. Vadose Zone Journal, 7(1):137-139, 2008a.

S. Lambot, E. C. Slob, D. Chavarro, M. Lubczynski, and $\mathrm{H}$. Vereecken. Measuring soil surface water content in irrigated areas of southern Tunisia using fullwaveform inversion of proximal GPR data. Near Surface Geophysics, 6:403-410, 2008b.

J. Ledieu, P. De Ridder, P. De Clercq, and S. Dautrebande. A method of measuring soil moisture by time domain reflectometry. Journal of Hydrology, 88:319-328, 1986.

M. C. Looms, K. H. Jensen, A. Binley, and L. Nielsen. Monitoring unsaturated flow and transport using cross-borehole geophysical methods. Vadose Zone Journal, 7(1):227-237, 2008.

I. A. Lunt, S. S. Hubbard, and Y. Rubin. Soil moisture content estimation using ground-penetrating radar reflection data. Journal of Hydrology, 307(1-4):254269, 2005.

J. Minet, S. Lambot, E.C. Slob, and M. Vanclooster. Soil surface water content estimation by full-waveform GPR signal inversion in the presence of thin layers. IEEE Transactions on Geoscience and Remote Sensing, In Press, 2010.

J. D. Redman, J. L. Davis, L. W. Galagedara, and G. W. Parkin. Field studies of GPR air launched surface reflectivity measurements of soil water content. In Steven Koppenjan and Lee Hua, editors, Proceedings of the Ninth International Conference on Ground Penetrating Radar, pages SPIE 4758:156-161, Santa Barbara, California, USA, 2002.

J. D. Rhoades, P. A. C. Raats, and R. J. Prather. Effects of liquid-phase electrical conductivity, water content, and surface conductivity on bulk soil electrical conductivity. Soil Science Society of America Journal, 40:651-655, 1976.
M. G. Schaap, D.A. Robinson, S.P. Friedman, and A. Lazar. Measurement and modeling of the TDR signal propagation through layered dielectric media. Soil Science Society of America Journal, 67: 1113:1121, 2003.

G. Schumann, D. J. Lunt, P. J. Valdes, R. A. M. de Jeu, K. Scipal, and P. D. Bates. Assessment of soil moisture fields from imperfect climate models with uncertain satellite observations. Hydrology and Earth System Sciences, 13(9):1545-1553, 2009.

G. Serbin and D. Or. Near-surface water content measurements using horn antenna radar: methodology and overview. Vadose Zone Journal, 2:500-510, 2003.

G. Serbin and D. Or. Ground-penetrating radar measurement of crop and surface water content dynamics. Remote Sensing of Environment, 96:119-134, 2005.

A. Stevens, B. van Wesemael, H. Bartholomeus, D. Rosillon, B. Tychon, and E. Ben-Dor. Laboratory, field and airborne spectroscopy for monitoring organic carbon content in agricultural soils. Geoderma, 144(1-2):395-404, 2008.

C. Strobbia and G. Cassiani. Multilayer groundpenetrating radar guided waves in shallow soil layers for estimating soil water content. Geophysics, 72(4): J17-J29, 2007.

J. van der Kruk. Properties of surface waveguides derived from inversion of fundamental and higher mode dispersive GPR data. IEEE Transactions on Geoscience and Remote Sensing, 44(10):2908-2915, 2006.

M. T. van Genuchten. A closed-form equation for predicting the hydraulic conductivity of unsaturated soils. Soil Science Society of America Journal, 44: 892-898, 1980.

H. Vereecken, J. A. Huisman, H. Bogena, J. Vanderborght, J. A. Vrugt, and J. W. Hopmans. On the value of soil moisture measurements in vadose zone hydrology: A review. Water Resources Research, 44, 2008.

N. E. C. Verhoest, H. Lievens, W. Wagner, J. AlvarezMozos, M. S. Moran, and F. Mattia. On the soil 
roughness parameterization problem in soil moisture retrieval of bare surfaces from synthetic aperture radar. Sensors, 8(7):4213-4248, 2008.

W. Wagner, G. Lemoine, and H. Rott. A method for estimating soil moisture from ERS scatterometer and soil data. Remote Sensing of Environment, 70(2): 191-207, 1999.

W. Wagner, G. Bloschl, P. Pampaloni, J. C. Calvet, B. Bizzarri, J. P. Wigneron, and Y. Kerr. Operational readiness of microwave remote sensing of soil moisture for hydrologic applications. Nordic Hydrology, 38(1):1-20, 2007.

L. Weihermüller, J. A. Huisman, S. Lambot, M. Herbst, and $\mathrm{H}$. Vereecken. Mapping the spatial variation of soil water content at the field scale with different ground penetrating radar techniques. Journal of $\mathrm{Hy}$ drology, 340:205-216, 2007.

E. Zehe, R. Becker, A. Bardossy, and E. Plate. Uncertainty of simulated catchment runoff response in the presence of threshold processes: Role of initial soil moisture and precipitation. Journal of Hydrology, 315(1-4):183-202, 2005. 